\title{
Implementation of Supervised Learning towards Optimizing Queries in Database Systems
}

\author{
Zdzislaw Polkowski, Mohanty Anita, Mishra Sambit Kumar
}

\begin{abstract}
Machine learning is a technology which with accumulated data provides better decisions towards future applications. It is also the scientific study of algorithms implemented efficiently to perform a specific task without using explicit instructions. It may also be viewed as a subset of artificial intelligence in which it may be linked with the ability to automatically learn and improve from experience without being explicitly programmed. Its primary intention is to allow the computers learn automatically and produce more accurate results in order to identify profitable opportunities. Combining machine learning with $A I$ and cognitive technologies can make it even more effective in processing large volumes human intervention or assistance and adjust actions accordingly. It may enable analyzing the huge data of information. It may also be linked to algorithm driven study towards improving the performance of the tasks. In such scenario, the techniques can be applied to judge and predict large data sets. The paper concerns the mechanism of supervised learning in the database systems, which would be self driven as well as secure. Also the citation of an organization dealing with student loans has been presented. The paper ends discussion, future direction and conclusion.
\end{abstract}

Keywords : Join enumeration, Join optimization, Query plan, Supervised learning, Symbolic learning

\section{INTRODUCTION}

In general, database management system manages big amounts of data along with data intensive applications. In this scenario, the data repository is maintained to build machine learning system and to improve the throughput. It is really expensive to generate query plans for the existing linked database management systems. Accordingly, the old execution plans can be reused to execute new queries which may be obtained in two phases. In the initial phase, representation of textual data linked to query plans are utilized to build the features extractor system. After that a solution mechanism is adopted towards query similarities. Then to build the system along with application of machine

Revised Manuscript Received on December 30, 2019.

* Correspondence Author

Zdzislaw Polkowski*, Wroclaw University of Economics and Business, Poland, E_Mail : zdzislaw.polkowski@ue.wroc.pl Institute of Technology, Cuttack, Affiliated to Biju Patnaik Uniersity of Technology, Rourkela, Odisha, India, E_Mail anitamohanty56@gmail.com

Sambit Kumar Mishra, Department of Computer Sc. \& Engg, Gandhi Institute for Education and Technology, Baniatangi, Affiliated to Biju Patnaik Uniersity of Technology, Rourkela, Odisha, India, Email: sambitmishra@gietbbsr.com

(C) The Authors. Published by Blue Eyes Intelligence Engineering and Sciences Publication (BEIESP). This is an open access article under the CC BY-NC-ND license (http://creativecommons.org/licenses/by-nc-nd/4.0/)
Mohanty Anita, Department of Computer Sc. \& Engg., Ajay Binay

learning techniques, the query execution plans are used along with classification based rules. In the present scenario, the machine learning may be used towards predictive analytics, typically in large volumes of data. The data management tools, embedding with machine learning, intelligence may have certain objectives like automation, optimization along with storage management. Using the modernizing tools, the data are categorized implementing the machine learning techniques and enabling the proper analytical correlations.

\section{REVIEW OF LITERATURE}

Shhab et al.[1] in their work have focused on growth and automation of large data sets. It is so important to maintain the data towards its usability. In such case, they suggested some effective machine learning algorithms to use the data in an effective manner.

Liao et al.[2] in their paper have projected towards data centers. In this case, the unsupervised learning sometimes may help to figure out which machines tend to work together, so that if those machines are put together or if there is some crisis, then the data centers can work more efficiently.

Haider et al. [3] in their work have discussed that the unsupervised Machine learning algorithms can also recognize the objects, class within a user's circle along with proper categorization.

Pang et al. [4] in their work have analyzed the representation of textual data as well as semantic data. Generally, the textual data may capture the facts and information, but it mostly fails to capture the sentiments and by that may lead to the misinterpretation of the true essence of the words. But somehow the sentiment analysis may be challenging and update with the ever complex use of statements. According to the author sentiment classification is helpful towards business intelligence applications.

Al-Hmouz et al. [5] in their work they discuss about mobile learning. They write, that in typical e-learning where there is wastage of bandwidth. In this technology information is accessed without many difficulties when desired to the mobile or portable devices. So, machine learning would drive the learning process of different users by providing information, which is customized to the preferences of the user. Wiese et al. [6] in their work have discussed about detection of unusual sequences of transactions, detection of unusual patterns of sensor. They have also focused about the dynamic machine learning method towards sequencing the operations. Kumar et al. [7] in their work have discussed about the technique of detection of predetermined patterns. In this case, machine learning may enable examination of the network traffic with predefined signatures along with updated databases. 
M. Raasveldt et al. [8] in their work have proposed the operations 1 linked a column-store databases. As being observed, it may give permission to achieve the merits of storage of data in the database implementing the analytical tools. The importance of the system can be linked to implementation of machine learning models directly in the database towards the use of the system along with metadata. The queries associated with the same may be applied towards training data sets.

T M. Raasveldt et al. [9] in their work have focused on the problems to be formulated using relational database management technologies. However, it has traditionally been very difficult to combine analytical tools with relational databases. The standard approach of running a separate database server and connecting to it through a socket connection is very inefficient and introduces severe bottlenecks when working with large amounts of data.

M. Vartak et al. [10] in their work have focused towards ModelDB system useful towards storing, tracking and managing machine learning models in their native environment. It may permit data, scientists to queries based on their metadata and quality metrics and also train and test data sets for each system. Also, it may not be linked to the mechanisms towards machine learning applications.

Shiqiang Wang et al. [11] in their work have focused towards learning protocol meant towards coordination and security. They were planning to incorporate the techniques linked to a generic class of machine learning along with trained data sets.

Miran Kim et al. [12] in their work have discussed regarding homomorphic encryption, which can be used to protect user data privacy through the parameter exchange under the encryption mechanism during machine learning. They have also studied regarding the possibility of leakage at the raw data level.

Reza Shokri et al. [13] in their research work have focused towards exchange of horizontally partitioned data along with training of neural networks by applying deep learning mechanisms.

Virginia Smith et al. [14] in their research paper have proposed the basic mechanism linked to multi-task style federated learning system that is used to permit multiple sites to complete separate tasks, while sharing knowledge and preserving security.

Raad Bahmani et al.[15] in their work have tried to define vertical federated learning with simplified mechanisms. As per the consideration, towards data privacy and security reasons, data may not be exchanged directly. In order to ensure the confidentiality of the data during the training process, a mechanism should be adopted regarding the same.

Rajesh Bordawekar et al. [16] in their work have tried to implement an unsupervised neural network based approach from Natural Language Processing to extract latent knowledge from the databases. The generated word-embedding model capture the semantic relationships between database tokens associated with contextual semantic relationships. It integrates the system and enable the analytical queries with semantic matching.

\section{TECHNIQUES ASSOCIATED WITH MACHINE LEARNING}

Machine learning is a data research technique that, by processing existing data, is able to predict future behaviors, results and trends. Computers learn without explicit programming using machine learning techniques. Machine learning solutions are iteratively compiled and have different phases:

- Data preparation

- Testing and teaching of models

- Implementing trained models

- Management of implementing models [17].

In general, with machine learning are associated the supervised and unsupervised techniques. Accordingly, the supervised machine learning technique is linked with new data sets to analyze the future occurrence and to evaluate the output values. In this regard, the algorithm related to the same may check the appropriateness of the outcomes. Similarly, considering the unsupervised machine learning techniques, it has been observed its non association with the classification, but somehow may be inclined to define the hidden structure of datasets not being properly synchronized. In such cases, the datasets may define the uncovered structures along with proper inference mechanisms.

Sometimes, it is seen that while implementing both supervised and unsupervised learning it may be a primary concern to enhance learning accuracy by adopting relevant resources with trained data sets. In most of the cases, the machines and software agents may determine the behavior of the system and try to maximize the system performance.

\section{A. Supervised Machine Learning}

While implementing supervised machine learning, initially the set of instructions can be trained along with predefined set of training methodologies to facilitate accuracy. In many applications of supervised learning, the main aim is to design and program a suitable inherited predictor function. In the similar way, the learning may be associated with mathematical algorithms to optimize the algorithm.

Particularly, the intention of machine learning is not to make accurate assumption, as it is linked to the domains where the initial guesses may be more profitable.

\section{B. Categorization In Supervised Machine Learning}

There are two primary categories linked towards supervised machine learning.

- Regression machine learning systems: In this case, the predicted value is directly involved in the continuous spectrum.

- Classification machine learning systems: In such case, the prediction mechanism is implemented with choice. In classification, the regression predictor is not useful.

\section{Unsupervised Machine Learning}

In case of unsupervised machine learning, it is primarily tasked with evaluating the link within the datasets. There may be no training examples implemented in this activity. The system is associated with a predefined dataset and tasked with finding patterns and matching. In general, this learning scheme is associated with a set of instructions along with huge set of data to find patterns and relationships. 


\section{ROLE OF MACHINE LEARNING IN THE DESIGN AND IMPLEMENTATION OF DATABASE SYSTEM}

The role of machine learning in designing database system could be to optimize the queries associated with the core database in which the database system may find the best physical execution path for structured queries.

In this work, the optimization techniques towards datasets in the databases are applied to understand the execution strategies along with different query plans. In fact, the statistical inference mechanisms are carefully balanced by applying generalization and update. While optimizing the join queries along with the learning mechanisms, is observed that the join enumeration techniques can be linked to sequential decision processes. In such cases, the join optimization may be used to select join operations to evaluate the structured queries. Sometimes, the learning mechanisms provide unique ideas to minimize sequential planning towards statistical implementation. The sub query plans can be treated as trained datasets to develop the system and may be focused towards upcoming instances by minimizing the complexity. The optimizer associated with the database queries may produce query plans that may exhibit intermediate results. The associated operations in this case are simply additional join types. The system after application of learning mechanisms would be appropriate to evaluate the advantages of storing as well as maintaining the datasets.

In many cases, the rule based system with decision and learning mechanisms may be useful towards representation and retrieval of data. The accuracy and appropriateness of the mechanism may directly or indirectly reflect towards the quality of the data supplied. In this regard, the unstructured datasets may select a signified subset of features of data sets. Accordingly, diversity is also be obtained while satisfying a single query, i.e., for single query it is quite difficult to compare ranking scores across collections as each one is unique in nature.

It has been observed that the mechanisms associated with the data retrieval approach are linked to symbolic learning based on a finite set of attributes. As the datasets in the databases reflect the frequencies with which terms appear, the data are assumed similar towards the databases in the server space. Each term is weighted by the number of query terms in the database so that a single appearance of the counts far less than a single appearance. The components of the term vector are not simply term frequencies, but term frequencies may be divided by the number of query terms in the databases in which that term appears. After that, the data linked to the databases are normalized. To measure the performance, the query terms, databases along with the weight of each data in the database is evaluated.

In many cases, the mechanisms can be adopted to implement a sequence grouping of strings in the database in terms of dividing the datasets into shorter strings. Accordingly, to compress the datasets, the indices as well as the data may be specified with proper citation. The compression mechanisms could be used to categorize the datasets in an effective manner to acquire maximum benefits. As a result, the data sets from each database can be implemented to maintain the process. Also the relative degrees of compression achieved by each dataset may be used to rank the categories. Considering the mechanisms, it is also required to extract the vital portion of the databases i.e. implementation of single words. In such cases, it is required to identify semantically significant phrases in databases consisting of datasets in keyword, name, title, and subject fields as well as certain patterns in the databases. Accordingly, each dataset can be rechecked for the complete set of phrases. While retrieving the data or data set from the database, the consistency should be maintained. Accordingly, the queries are required to be satisfied and the accountability must be maintained by their data servers along with the retrieval strategies. Considering the grouped training queries, the retrieval mechanism of query terms may depend on the weighting factor of each group and required to be matched with a similar group of databases. While categorizing the data within the data servers, synchronization with labeling is essential. In such scenario, automating the tasks linked to classification may be essential. As soon as the system may achieve a certain level of accuracy, the performance of tasks will be easier. Again, it must be consistent. Sometimes, the users classify the data with inconsistently due to adoption of fuzzy boundaries in the classification schemes, the performance in this regard may be improved to attain maximum outputs at this level. In many cases, it is quite natural to adopt queries linked to natural language. Sometimes these are required to be converted to Boolean queries. To accomplish these activities, it can be required to make the process iterative. It has clear that the machine learning techniques would be applied to user modeling, feedback as well as data filtering while formulating queries.

\section{SUPPORT VECTOR MACHINE}

The Supporting Vector Machines (SVM) method offers very high accuracy compared to other classifiers, such as logistic regression and decision trees. The advantage of SVM is its use of nonlinear data. It is used in various applications face detection, intrusion detection, e-mail, press articles and website classification, gene classification and handwriting recognition. SVM can easily handle many continuous and categorical variables. SVM constructs a hyperplane in multidimensional space to separate different classes. SVM generates an optimal hyperplane in a repeatable manner that is used to minimize error. The basic idea of SVM is to find the maximum edge hyperplane (MMH) that best divides the data set into classes. SVM can be used for both classification and regression challenges. However, it is most often used in classification problems [18]. Thus, the support-vector machine may be cited as a system associated with supervised learning and accordingly the algorithm may be developed to analyze data towards classification. But it may work consistently on nonlinear classification systems. Sometimes during unavailability of data, it may evaluate natural cluster of data and try to form new datasets with proper categorizations. Also, it can be useful towards textual data along with virtual data in real time application to minimize the anomalies with the data sets. 
In general, it could be defined as a pattern classifier associated with statistical learning technique to eradicate the bugs maintains the margin between the virtualized data with the data servers. In such case, it may maximize the limit associated with hyperplane and data.

\section{PROBLEM FORMULATION WITH IMPLEMENTATION}

The mechanism of supervised learning in the application of database can be self driven as well as secure. And to some extent may be useful to eradicate errors occurred in the database management system operated manually. Also, it may have the capability of self explanatory as well as decision making.

\section{A. The Citation Of An Organization Dealing With Student Loans To Meritorious Students}

Problem statement: Company Dummy_Studsys may want to automate the eligibility of loan scheme linked to the details like Student_ID, Name, Education, CGPA, Amount. Accordingly, it is required to acquire the relevant data and make some application of machine learning to automate the decision making process. Applications using the machine learning platform can be responsible towards creating and sharing the relevant and requisite data. Accordingly implementing the structured query language, including data definition, data manipulation the capabilities of machine learning may be evaluated along with the trained and predicted data.

There are some sequential steps implementing the structured query language to process directly on the input as well as predicted data as follows.

\section{ALGORITHM}

I. Creation of Database to be stored in the Data warehouse along with Data insertion CREATE TABLE STUDSYS

(STUDENT_ID VARCHAR2(20),

NAME VARCHAR2(20),

EDUCATION VARCHAR2(20),

CGPA NUMBER $(7,2)$,

AMOUNT NUMBER(7));

II. Insert Data into the database after creation of the database

\section{INSERT INTO STUDSYS} \&CGPA, \&AMOUNT);

III. Activate the Machine Learning Process to recognize the pattern in the current data set towards dealing with student loan by linking with machine learning user interface along with the database.

IV. Analyze the database by applying machine learning to verify the approval of student loans by applying Data manipulation operations

V. Split and enumerate the dataset into training dataset as well as test data set. Accordingly the two databases may be created by using data definition language

CREATE TABLE STUDSYS_TRAIN_DATA AS SELECT

* FROM STUDSYS SAMPLE (20);
VALUES('\&STUDENT_ID','\&NAME','\&EDUCATION',

CREATE TABLE STUDSYS_TEST_DATA AS SELECT * FROM STUDSYS MINUS SELECT * FROM

STUDSYS_TRAIN_DATA;

VI. Prepare classification mechanism implementing machine learning

BEGIN

EXECUTE IMMEDIATE 'CREATE TABLE

STUDSYS_SETTINGS (SETTING_NAME

VARCHAR2(20), SETTING_VALUE NUMBER(9))';

EXECUTE IMMEDIATE 'INSERT INTO

STUDSYS_SETTINGS (SETTING_NAME,

SETTING VALUE) VALUES ('\&NAME', \&VALUE)';

EXECUTE IMMEDIATE 'CALL

DBMS DATA MINING.CREATE SYS('STUDSYS SYS

','STUDSYS','STUDSY TRAIN DATA','AMOUNT',',S

TUDSYS SETTINGS')';

END;

VII. Use the Database triggers to test the quality of the system to the test dataset. While checking the data to the dataset, it may be predicted the authenticity of the student loan approval.

ALTER TABLE STUDSYS_TEST_DATA ADD

STUDYLOAN_STATUS VARCHAR2(7);

UPDATE STUDYLOAN_TEST_DATA SET

$L O A N \_E L I G I B I L I T Y=P R E D(S T U D S Y S$ LOAN_CLASS

$U S I N G *)$;

To check the accuracy towards a decision

SELECT TO_CHAR(((SELECT COUNT(*) FROM

STUDSYS_TEST_DATA WHERE LOAN_ELIGIBILITY

= LOAN_ELIGIBILITY_PRED1)/(SELECT COUNT ${ }^{*}$ )

FROM STUDYLOAN_TEST_DATA))*100,'999.99')

CORRECT_PRED_PERCENTAGE FROM DUAL;

In addition to evaluate the status and make accurate prediction towards test dataset by applying machine learning and grouping attributes

SELECT STUDYLOAN_STATUS,

STUDYLOAN_STATUS_PRED, COUNT(*) from

STUDSYS_TEST_DATA GROUP BY

STUDYLOAN_STATUS, STUDYLOAN_STATUS_PRED

ORDER BY 1,2 may be evaluated.

Implementation Mechanism

Step 1 : Define database to store data def save_in_database(studsys_data)

filename=input( Database(studsys_data))

Step2 : Import mysql

Step3 : conn=mysql.connect(str(studsys))

$$
\text { cur=conn.cursor() }
$$

Step 4 : cur.execute('create table if not exists moveinfo(STUDENT_ID VARCHAR2(20), NAME VARCHAR2(20), EDUCATION VARCHAR2(20),

CGPA NUMBER(7,2), AMOUNT

$\left.\operatorname{NUMBER}(7)^{\prime}\right)$

cur.execute('select * from moveinfo where

amount $=20000$ ', student_id)

row $=$ cur.fetchone ()

Step 5 : Implement the mechanism to separate the set of training data sets to individual categories. $\mathrm{t}=\left\{\mathrm{x}_{\mathrm{i}}, \mathrm{y}_{\mathrm{i}}\right)$, where $\left.\mathrm{i}=1 \ldots \ldots \mathrm{n}\right\}$ along with a hyper

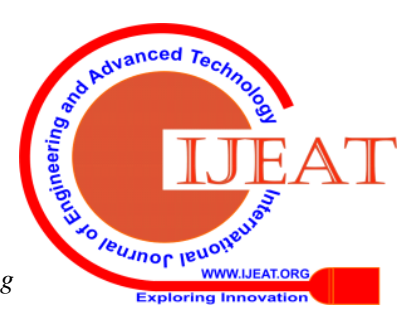


plane $\mathrm{q}^{\mathrm{T}} \mathrm{a}(\mathrm{x})+\mathrm{b}=0$, where $\mathrm{x}_{\mathrm{i}}=$ ith input vector, $\mathrm{y}_{\mathrm{i}}=$ targeted binary vector

Step 6 : Satisfying the conditions by the classifier

a. $\mathrm{q}^{\mathrm{T}} \mathrm{a}(\mathrm{x})+\mathrm{b}>=1$ provided $\mathrm{yi}=1$

b. $\mathrm{q}^{\mathrm{T}} \mathrm{a}(\mathrm{x})+\mathrm{b}<=1$, provided $\mathrm{yi}=-1$

Mapping the input to desired featured space may be linearly separable.

Step 7 : Obtain the coverage of path of training data sets, by initiating the loop

$$
\mathrm{i}=1 \text {; }
$$

while $(\mathrm{i}<=\mathrm{n})$

$\operatorname{cpt}(\mathrm{i})=\left(\mathrm{q}^{\mathrm{T}} \mathrm{a}(\mathrm{x}(\mathrm{i}))+\mathrm{b}\right) / \mathrm{q} * \mathrm{i}$;

$\mathrm{i}++$;

\}
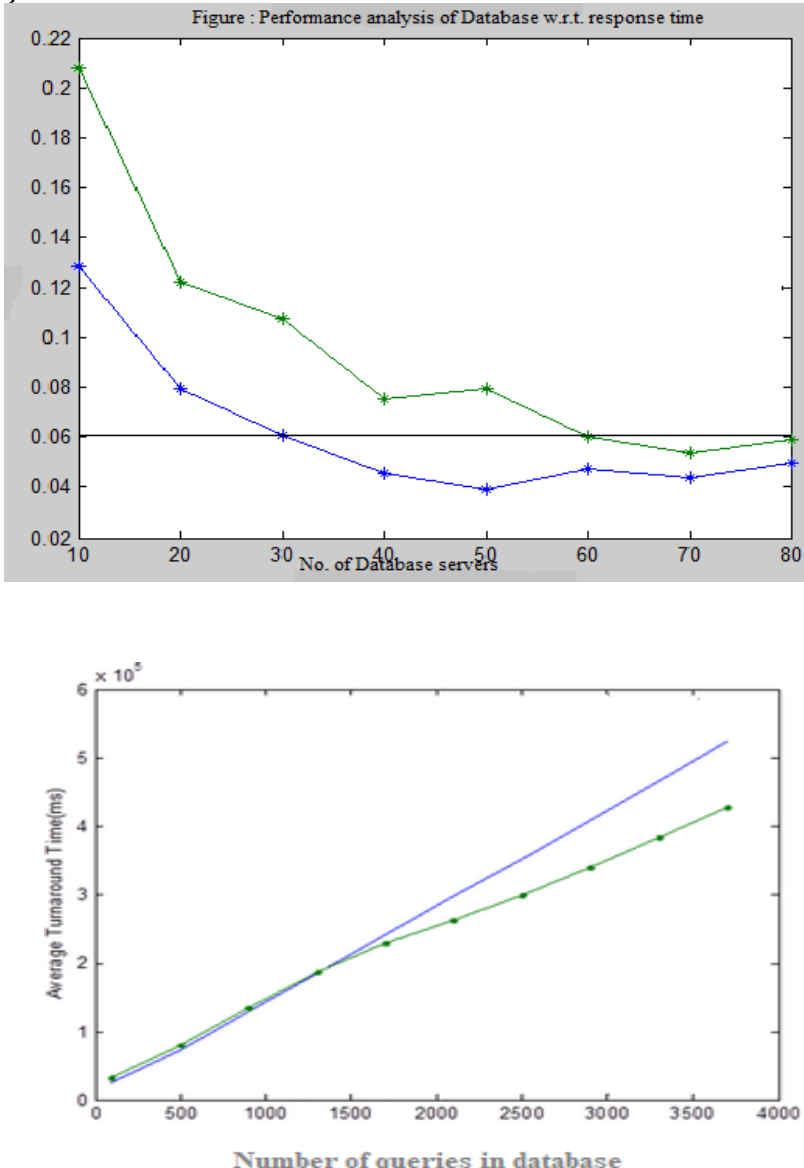

Table I-: Analysis of Performance of Database Server w.r.t. response time

\begin{tabular}{|c|c|c|c|c|}
\hline $\begin{array}{c}\text { Number } \\
\text { of } \\
\text { Database } \\
\text { Servers }\end{array}$ & $\begin{array}{c}\text { Number of } \\
\text { queries } \\
\text { associated } \\
\text { with the } \\
\text { database }\end{array}$ & $\begin{array}{c}\text { Number of } \\
\text { plans } \\
\text { associated } \\
\text { with the } \\
\text { queries }\end{array}$ & $\begin{array}{c}\text { Processor } \\
\text { response } \\
\text { time(ms) }\end{array}$ & $\begin{array}{c}\text { Average } \\
\text { turn } \\
\text { around } \\
\text { time } \\
\text { (waiting } \\
\text { time+ } \\
\text { burst } \\
\text { time) } \\
\text { (ms) }\end{array}$ \\
\hline 10 & 500 & 500 & 0.13 & 0.65 \\
\hline 20 & 1000 & 500 & 0.079 & 1.47 \\
\hline 30 & 1500 & 500 & 0.06 & 2.23 \\
\hline 40 & 2000 & 500 & 0.05 & 2.45 \\
\hline 50 & 2500 & 500 & 0.047 & 2.79 \\
\hline 60 & 3000 & 500 & 0.045 & 3.13 \\
\hline 70 & 3500 & 500 & 0.41 & 3.47 \\
\hline
\end{tabular}

The mechanisms associated with this perform better along with the limited training set. Accordingly, several executions provide the same results towards different parametric variables. The classification in this case may define the feature space and the capacity of classification may not be a large size domain. Also, it is noted that the approach towards achieving solution mechanism of queries may maintain the semantic structure along with encapsulation of semantic clustering and analogy sequences. The provision to manipulate the data and implementation of queries will develop strong semantic relationships within the data files as well as in the databases. During the initialization process, the data will be converted from sequenced data frame to memory databases. In case, obtaining special case of similarity, the query plans will first be chosen along with similarity index of datasets.

\section{EXPERIMENTAL ANALYSIS}

The classification in this case is defined with the feature space and the capacity of classification is not a large size domain.

\begin{tabular}{|l|l|l|}
\hline $\begin{array}{l}\text { No. of queries } \\
\text { with database }\end{array}$ & $\begin{array}{l}\text { No. of plans } \\
\text { with queries }\end{array}$ & $\begin{array}{l}\text { Response } \\
\text { time(ms) }\end{array}$ \\
\hline 1500 & 500 & 0.06 \\
\hline 2000 & 500 & 0.05 \\
\hline 2500 & 500 & 0.047 \\
\hline 3000 & 500 & 0.045 \\
\hline
\end{tabular}

It is noted that the approach towards achieving solution mechanism of queries maintains the semantic structure along with encapsulation of semantic clustering and analogy sequences. Accordingly, implementation of the data as well as queries will develop strong semantic relationships within the data files as well as in the databases.

\section{DISCUSSION AND FUTURE DIRECTION}

The analysis of complex query plans while linking to a column-store databases, performance towards training, testing and prediction can be evaluated implementing the techniques associated with machine learning. In some cases, there are unnecessary overhead in the serialization of the system. Accordingly, linking the system with the database, it can be evaluated along with its performance. Accordingly, the system could be approached directly towards memory representation to avoid this type of overhead. It is also required to focus on out-of-memory datasets of heterogeneity.

It is worth mentioning that in the future dynamic development of Automatic databases can be expected. The latest and most breakthrough databases, automatic databases (also known autonomous databases), operate in the cloud and use machine learning to automate optimization, security, backup and updating of databases, and other routine management tasks traditionally performed by database administrators. These areas seem to be interesting from a scientific point of view and can set the direction for further development of the use of machine learning in database management systems.

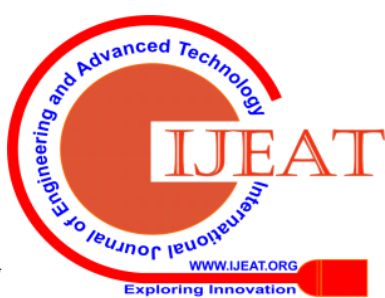




\section{CONCLUSION}

While analyzing the performance of machine learning towards implementation of queries in a database, it has been projected to specify the tasks linked to classification along with an assigned load to the database servers. The results obtained with respect to the response time may be better as compared to other conventional approaches. It has been observed that the test results may be linked to better generalization.

\section{REFERENCES}

1. Shhab, Areej, Gongde Guo, and Daniel Neagu. "A Study on Applications of Machine Learning Techniques in Data Mining." Proc. of the 22nd BNCOD workshop on Data Mining and Knowledge Discovery in Databases, Sunderland, UK. 2005.

2. Liao, Shih-wei, et al. "Machine learning-based prefetch optimization for data center applications." Proceedings of the Conference on High Performance Computing Networking, Storage and Analysis. ACM, 2009.

3. Haider, Peter, Luca Chiarandini, and Ulf Brefeld. "Discriminative clustering for market segmentation." Proceedings of the 18th ACM SIGKDD international conference on Knowledge discovery and data mining. ACM, 2012.

4. Pang, Bo, Lillian Lee, and Shivakumar Vaithyanathan. "Thumbs up? sentiment classification using machine learning techniques." Proceedings of the ACL-02 conference on Empirical methods in natural language processing-Volume 10. Association for Computational Linguistics, 2002.

5. Al-Hmouz, Ahmed, Jun Shen, and Jun Yan. "A machine learning based framework for adaptive mobile learning." Advances in Web Based Learning-ICWL 2009. Springer Berlin Heidelberg, 2009. 34-43.

6. Wiese, Bénard, and Christian Omlin. Credit card transactions, fraud detection, and machine learning: Modelling time with LSTM recurrent neural networks. Springer Berlin Heidelberg, 2009.

7. Kumar, Vinod, and Dr Om Prakash Sangwan. "Signature Based Intrusion Detection System Using SNORT." International Journal of Computer Applications \& Information Technology 1 (2012).

8. M. Raasveldt and H. Mühleisen. Vectorized UDFs in Column-Stores. In Proceedings of the 28th International Conference on Scientific and Statistical Database Management, SSDBM 2016, Budapest, Hungary, July 18-20, 2016, pages 16:1-16:12, 2016

9. M. Raasveldt and H. Mühleisen. Don't Hold My Data Hostage: A Case for Client Protocol Redesign. Proc. VLDB Endow., 10(10):1022-1033, June 2017.

10. M. Vartak, H. Subramanyam, W.-E. Lee, S. Viswanathan, S. Husnoo, S. Madden, and M. Zaharia. Model DB: a system for machine learning model management. In Proceedings of the Workshop on Human-In-the-Loop Data Analytics, page 14. ACM, 2016.

11. Shiqiang Wang, Tiffany Tuor, Theodoros Salonidis, Kin K. Leung, Christian Makaya, Ting He, and Kevin Chan. 2018. When Edge Meets Learning: Adaptive Control for Resource-Constrained Distributed Machine Learning. CoRR abs/1804.05271 (2018). arXiv:1804.05271 http://arxiv.org/abs/1804.05271.

12. Miran Kim, Yongsoo Song, Shuang Wang, Yuhou Xia, and Xiaoqian Jiang. 2018. Secure Logistic Regression Based on Homomorphic Encryption: Design and Evaluation. JMIR Med Inform 6, 2 (17 Apr 2018), e19. https://doi.org/10.2196/ medinform.8805.

13. Reza Shokri and Vitaly Shmatikov. 2015. Privacy-Preserving Deep Learning. In Proceedings of the 22Nd ACM SIGSAC Conference on Computer and Communications Security (CCS '15). ACM, New York, NY, USA, 1310-1321. htps: //doi.org/10.1145/2810103.2813687

14. Virginia Smith, Chao-Kai Chiang, Maziar Sanjabi, and Ameet S Talwalkar. 2017. Federated Multi-Task Learning. In Advances in Neural Information Processing Systems 30, I. Guyon, U. V. Luxburg, S. Bengio, H. Wallach, R. Fergus, S. Vishwanathan, and R. Garnett (Eds.). Curran Associates, Inc., 4424-4434. http://papers.nips.cc/paper/ 7029-federated-multi-task-learning.pdf.

15. Raad Bahmani, Manuel Barbosa, Ferdinand Brasser, Bernardo Portela, Ahmad-Reza Sadeghi, Guillaume Scerri, and Bogdan Warinschi. 2017. Secure Multiparty Computation from SGX. In Financial Cryptography and Data Security - 21st International Conference, FC 2017, Sliema, Malta, April 3-7, 2017, Revised Selected Papers. 477-497. https: //doi.org/10.1007/978-3-319-70972-7_27.
16. Rajesh Bordawekar, Bortik Bandyopadhyay, and Oded Shmueli. 2017. Cognitive Database: A Step towards Endowing Relational Databases with Artificial Intelligence Capabilities. CoRR abs/1712.07199 (December 2017). http: //arxiv.org/abs/1712.07199.

17. Microsoft, Jakie produkty z zakresu uczenia maszynowego oferuje firma Microsoft? Available :

https://docs.microsoft.com/pl-pl/azure/architecture/data-guide/technol ogy-choices/data-science-and-machine-learning, accessed November 2019

18. Enroute, Klasyfikacja metoda wektorów nośnych SVM. Available at: http://enroute.pl/klasyfikacja-metoda-wektorow-nosnych-supportingvector-machines-svm/, accessed November 2019

\section{AUTHORS PROFILE}

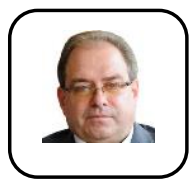

Dr. Zdzislaw Polkowski is presently associated with Wroclaw University of Economics and Business, Poland, He holds a $\mathrm{PhD}$ degree in Computer Science and Management from Wroclaw University of Technology, Post Graduate degree in Microcomputer Systems in Management from University of Economics in Wroclaw and Post Graduate degree IT in Education from Economics University in Katowice. He obtained his Engineering degree in Industrial Computer Systems from Technical University of Zielona Gora. He has published more than 55 papers in journals, 15 conference proceedings, including more than 8 papers in journals indexed in the Web of Science. He served as a member of Technical Program Committee in many International conferences in Poland, India, China, Iran, Romania and Bulgaria.

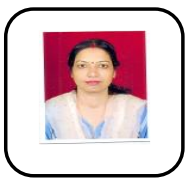

Mrs. Anita Mohanty is having more than 10 years of experience in different AICTE approved institutions. She is having good number of publications in different indexed Journals

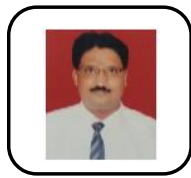

Dr.Sambit Kumar Mishra is having more than 22 years of experience in different AICTE approved institutions. He has more than 29 publications in different peer reviewed International Journals and editorial board member of different peer reviewed indexed Journals. 